\section{APLICAÇÕES DO LASER NA ACUPUNTURA}

\section{LASER APPLICATIONS IN ACUPUNCTURE}

\section{RESUMO}

A Acupuntura é uma técnica milenar e uma das terapias oriundas da Medicina Tradicional Chinesa (MTC). As técnicas da MTC são utilizadas em pontos específicos, por meio de inserção de agulhas, moxas, ventosas, massagens, estímulos elétricos e Laser. Esses estímulos promovem a circulação do Qi e sangue, aumentando o fluxo energético nos meridianos, melhorando assim, o equilíbrio Yin-Yang. $\mathrm{O}$ uso do Laser é uma alternativa para a realização da Acupuntura pois é um método indolor de estimulação dos pontos, este pode ser utilizado quando a técnica com as tradicionais agulhas não são indicadas. A grande vantagem da laserpuntura é não causar nenhuma agressão à pele evitando possíveis infecções, não causar dor podendo ser utilizada em pessoas mais sensíveis e em crianças, idosos e paciente com problemas mentais e em pessoas que apresentam problemas de coagulação. No presente estudo foi realizada uma busca por artigos científicos, livros, dissertações e teses de Acupuntura e Laserterapia. O objetivo é buscar na literatura uma padronização mínima de parâmetros necessários para o tratamento com Laser, como: comprimento de onda, potência do equipamento, tempo, dose utilizadas nos pontos, número de acupontos e número de sessões. Os resultados mostram uma escassez de material teórico-prático sobre o tema, controversas em resultados e com trabalhos publicados sem critério de execução. Conclui-se que protocolos sem nenhuma padronização nos são apresentados e parâmetros de aplicação são praticamente esquecidos nas discussões destes artigos. Este trabalho visa suprir esta necessidade, servindo como meio de informação e suporte para aqueles que necessitam de assistência neste âmbito.

\section{PALAVRAS-CHAVE}

Acupuntura.

Laser na Acupuntura.

Laser.

Medicina Tradicional Chinesa.

\section{Caroline Valente}

- Docente da Pós-Graduação em Acupuntura na Faculdades Integradas Espírita e Universidade Regional de Blumenau.

Fernanda de Lacerda Gomara

- Docente da Pós-Graduação em Acupuntura na Faculdades Integradas Espírita.

Pedro Lagos Marques Neto

- Coordenador da Pós-Graduação em Acupuntura na Faculdades Integradas Espírita. Docente da Pós-Graduação em Acupuntura no IBRATE, EBRAMEC E INSPIRAR

Regina Celia de Souza

- Docente da Pós-Graduação em Acupuntura na Soepar-Ceap-PR

\section{CORRESPONDENTE}

\section{Caroline Valente.}

Universidade Regional de Blumenau. R. Antônio da Veiga, 140 - Victor Konder, Blumenau - SC, 89012-900

E- M A I L

carolvalente11@gmail.com

Recebido: 25/04/2014

Aprovado: 03/04/2015 


\begin{abstract}
Acupuncture is an ancient technique and one of the therapies derived from Traditional Chinese Medicine (TCM). The techniques of TCM are used at specific body points through insertion of needles, moxa, cupping, massage, electrical stimulation and laser. These stimuli promote Qi and blood circulation, what increases the energy flow in the meridians and improves the Yin-Yang balance. The laser application is an alternative technique for Acupuncture because it is a painless method of point stimulation treatment and may be applied when traditional needle technique is not indicated. The great advantage of laser acupuncture is related to the fact that causes no damages to the skin, therefore, avoiding possible infections problems; it is painless and can be used in sensitive people, children, elderly, patients with mental health problems and people with clotting problems. In the present study, a search for scientific articles, books, dissertations and theses for Acupuncture and Laser Therapy was performed. The objective is to search for information of minimum standardization methods necessary for treatment with laser, considering parameters as wavelength, power equipment, time, dose used in points, number of acupoints and number of sessions. The results show a gap between theorical and practical material on the subject, controversial results and published works without implementation criteria. It is concluded that no standardization protocols are presented and applied parameters are practically forgotten in discussions on these articles. This work aims to fill this need, serving as a source of information and support for those who need assistance in this area.
\end{abstract}

KEYWORDS: Acupuncture, Laser Acupuncture, Laser, Traditional Chinese Medicine.

\section{INTRODUÇÃO}

A Acupuntura é uma técnica oriunda da MTC, ciência milenar, surgida na China antes de Cristo e consiste no estímulo de determinados pontos, sendo um método independente da Medicina Ocidental'. Atualmente a MTC é considerada "um método de tratamento complementar" de acordo com a Organização Mundial da Saúde (OMS) e faz parte da Política Nacional de Práticas Integrativas e Complementares (PNPIC) introduzida pelo Ministério da Saúde, por meio da Portaria GM no 971, de 03 de maio de $2006^{2}$. A MTC está baseada em princípios filosóficos, na observação dos fenômenos da natureza e sua influência energética no ser humano e em suas relações internas e externas ${ }^{3,4}$.

Dentro da MTC, a doença é considerada uma manifestação de desequilíbrio, e a Acupuntura (uma das práticas da MTC) seria uma forma de readquirir essa harmonia perdida. A Acupuntura baseia-se no fluxo harmônico (circulação) da energia Qi (também conhecida como energia vital) pelo organismo. Segundo alguns princípios da MTC, a saúde do individuo é determinada pela qualidade do Qi e do sangue que circula pelo corpo. Quando o Qi e o sangue circulam de forma equilibrada, o organismo funciona na sua plenitude ${ }^{3,5}$.Essa técnica permite o desbloqueio e circulação do Qi, promovendo a harmonização e fortalecimento dos órgãos, vísceras e do corpo humano como um todo ${ }^{1}$. Baseia-se em princípios filosóficos do TAO, na teoria dos Cinco Elementos, das energias YIN e YANG e dos Meridianos ou canais de energia ${ }^{3,6,5}$.

A Acupuntura consiste na estimulação de pontos específicos na pele com o objetivo de liberar neurotransmissores e outras substâncias ${ }^{7}$.Trata-se de uma técnica onde as agulhas são inseridas com o objetivo de estimular determinados pontos do corpo, denominados acupontos. Esses acupontos estão localizados por toda a superfície do corpo e apresentam maior condutibilidade elétrica devido a uma maior densidade das junções comunicantes (gap junctions) de suas células, além de apresentarem um aumento nas taxas metabólicas, na temperatura e na concentração de cálcio intracelular . Na orelha também existem pontos de Acupuntura e a prática dessa recebe o nome de Acupuntura auricular ${ }^{8,9}$.

As principais formas de estimulação dos pontos de Acupuntura são as próprias agulhas, digitopres- 
são (Do-In, Tuiná, Reflexologia), eletroacupuntura (estímulo elétrico), moxabustão (queima da erva Artemísia vulgaris) e Laser ${ }^{10,11}$.

A Laserpuntura é a união de duas técnicas, a Acupuntura que é utilizada há milênios e a Laserterapia que surgiu em $1970^{12}$. A palavra Laser vem da língua inglesa e significa: Light Amplification by Stimulated Emission of Radiation, cuja tradução seria: amplificação de luz por emissão estimulada de radiação. Esta radiação é eletromagnética e não ionizante, por isso não causa danos à saúde. Podemos trabalhar com o Laser de diversas formas, e aquela que tem indicação em Acupuntura é o estímulo do Laser em baixa intensidade de energia, ou também conhecido como Laser terapêutico' ${ }^{1}$.

A Laserpuntura é considerada uma prática importante, pois permite a estimulação dos pontos de Acupuntura e pode ser usado no tratamento das mais diversas patologias ${ }^{13}$. Entre as vantagens encontradas na Laserpuntura, podemos destacar: curta duração de aplicação (tempo de estímulo por acuponto pode variar de 30 segundos a 2 minutos), não causa câncer (não realça o crescimento de tumores), inofensivo à pele evitando possíveis infecções, indolor (apropriado para pessoas mais sensíveis à dor) e pode ser combinada com outros tratamentos. Por tanto é uma ótima opção para tratamento de pacientes sensíveis à dor, com fobias de agulhas, com processos dolorosos agudo, em crianças, idosos, pacientes psiquiátricos, em pessoas que apresentam problemas de coagulação ou estão utilizando medicamentos anticoagulantes e também pode ser utilizado na veterinária, pois é uma excelente alternativa para o tratamento de animais agressivos e filhotes ${ }^{1,6,12-16}$.

Também possui maior segurança e higiene, pois não causa sangramento durante o tratamento; evita-se contaminações por parte do profissional, que poderia contaminar-se ao remover as agulhas que tivessem gerado algum tipo de sangramento no paciente $e^{1,17}$.

O Laser traz muitos benefícios para o meio ambiente poisnão gera resíduos, ao passo que na Acupuntura as agulhas utilizadas nos pacientes precisam ser descartadas no meio ambiente de maneira correta, ou seja, é necessário um coletor de perfu- rocortantes, cujo destino será a incineração, nunca aterros sanitários ou lixões ${ }^{1}$.

Como todas as técnicas da MTC o Laser também possui algumas restrições com relação ao seu uso: não utilizar em gestantes; não irradiar diretamente sobre a retina, pois pode ocasionar lesões irreversíveis e perda de visão; não irradiar sobre massas neoplásicas ou em portadores de neoplasias, pois o Laser possui caráter bioestimulativo e pode aumentar a proliferação das células tumorais; não irradiar sobre focos de infecções bacterianas devido à possibilidade de crescimento e multiplicação dos microrganismos ${ }^{6,18}$.

As radiações utilizadas na Acupuntura pelos aparelhos de Lasers estão situadas entre os comprimentos de ondas da luz visível (400-700nm) e radiação infravermelha (IR) $(760 \mathrm{~nm}-1 \mathrm{~mm})$. Os efeitos bioestimuladores dos Lasers de baixa intensidade foram descritos por vários autores e esses efeitos foram divididos em indiretos, diretos e gerais. Os efeitos indiretos são a estimulação da circulação e da regeneração celular. Já os efeitos diretos, os bioquímicos, bioelétricos e bioenergéticos e os efeitos gerais possuem natureza anti-inflamatória, analgésica e anti-edematoso ${ }^{19-21}$.

Uma questão relevante para a Laserpuntura é se o Laser pode fazer o mesmo efeito da agulha, já que as agulhas podem penetrar na pele. Para responder a essa pergunta precisamos levar em conta alguns aspectos relacionados à pele e à constituição física de cada indivíduo, pois o grau de dispersão e absorção da irradiância incidente nos diferentes elementos do tecido devem ser considerados ${ }^{22,23}$. Epelbaum ${ }^{25}$ complementa que a porção de energia absorvida e transmitida não depende somente de parâmetros como intensidade e área de irradiação e comprimento de onda, mas também de fatores individuais como pigmentação da pele, idade e espessura do tecido alvo, o que parece influenciar o sucesso da terapia por Acupuntura a Laser.

A radiação incidente geralmente decai exponencialmente à medida que passa através da pele devido à sua interação com a mesma, respeitando as suas propriedades ópticas de reflexão ou de absorção. Algumas radiações podem penetrar superficialmente ou profundamente na pele. A radiação IR penetra 
mais profundamente na pele do que as radiações da faixa do ultravioleta (UV) ou visível, atingindo até a derme e podendo chegar aos tecidos subcutâneos ${ }^{22-24}$.

O conhecimento básico sobre Laser ainda é pouco difundido pelos profissionais da Acupuntura, principalmente por aqueles que não foram devidamente capacitados. Há necessidade de um aprofundamento e detalhamento nos estudos, uma vez que ainda falta padronização de metodologias e consenso sobre parâmetros como comprimento de onda, potência, dose, tempo de aplicação, quantidade e frequência dessas aplicações. Embora nos últimos anos alguns autores ${ }^{1,6,13,25}$ tenham pesquisado e desenvolvido protocolos de atendimento, a escassez de diretrizes norteadoras ainda gera controvérsia na aplicação do Laser em seu uso clínico.

Por todas essas divergências, ainda são necessárias muitas pesquisas e desenvolvimento de protocolos de atendimentos para alcançar uma padronização do uso do Laser na Acupuntura. Uma avaliação objetiva de sua eficácia em estudos publicados é difícil, pois os referidos parâmetros básicos de tratamento não estão detalhados. Faz-se necessário, assim, uma descrição mais aprofundada para fornecer subsídios à discussão do Laser na Acupuntura.

\section{METODOLOGIA}

Trata-se de uma revisão e foram utilizados dados obtidos a partir da literatura disponível. Para a coleta de dados foram utilizados livros, artigos, dissertações e teses de Acupuntura e Laserterapia. Os artigos foram pesquisados nos bancos de dados do PubMed e ScienceDirect, compreendidos no período de 1990 até 2014. Palavras de busca: Laser, Laser da baixa intensidade, Acupuntura, Laserpuntura e MTC.

O material científico foi lido e seus dados foram analisados e discutidos. Uma tabela foi elaborada com os parâmetros necessários para o tratamento com Laser, como: comprimento de onda utilizado, potência do equipamento, tempo utilizado no tratamento, dose utilizadas nos pontos de tratamento, número de acupontos e número de sessões de tratamento. Os parâmetros obtidos foram normatizados e uma tabela foi gerada para análise e respectiva discussão.

\section{RESULTADOS E DISCUSSÃO}

Por tratar-se de um tema com muitas divergências e sem uma padronização é importante ressaltarmos que foi levantada uma grande quantidade de artigos disponíveis sobre Laser e Laser na Acupuntura no período de tempo compreendido entre 1971 e 2014 nas bases de dados do PubMed e ScienceDirect.

Essa revisão bibliográfica contemplou quase 40 anos da história do Laser e do uso do Laser na Acupuntura. Nesse período observamos uma gama grande de estudos sobre o tema, entretanto, uma avaliação mais objetiva dos resultados obtidos é difícil, devido a vários fatores que causam confusão e controvérsia no entendimento desse estudo. Observamos que variáveis de primeira ordem muito importantes como comprimento de onda, irradiação, tempo, propriedades e espessura da pele e que são determinantes para os resultados são muitas vezes desconsideradas ou não citadas. A maioria dos trabalhos em que os autores concluíram fracasso no uso do Laser na Acupuntura ocorreu devido à baixa potência do equipamento utilizado no estudo, escolha incorreta do comprimento de onda ou simplesmente pelo uso inadequado dos parâmetros previamente escolhidos para a realização dos experimentos.

Muitos profissionais da Acupuntura têm lançado mão desta ferramenta em suas práticas clínicas, causando um impacto negativo no uso e na eficácia da técnica do Laser, entretanto o que se observa é uma falta de padronização sobre o tema, escassez na literatura, controversas em resultados e trabalhos publicados com baixo rigor científico e sem critério de execução.

Um dos grandes problemas encontrados é o desconhecimento sobre as características físicas de cada pessoa, pois a eficácia do Laser depende de alguns fatores importantes como os tecidos biológicos e a constituição física de cada paciente. Indivíduos de pele clara precisam de doses maiores que indivíduos de pele escura, regiões corpóreas onde a epiderme é mais espessa ou com maior camada de gordura, usa-se doses maiores, pois ao incidir sobre o tecido do paciente a radiação pode ser absorvida, refletida ou 
espalhada. Indivíduos em estado nutricional normal precisam de doses menores que indivíduos desnutridos e o estresse pode determinar a necessidade de doses maiores. Tais variáveis receberam pouca ou nenhuma atenção em qualquer projeto de estudos de Acupuntura à Laser.

Em resumo, as propriedades ópticas da pele e do tecido subjacente, em combinação com a quantida- de de radiação incidente, determinam a transmissão de luz. Tudo indica que a eficácia da Acupuntura à Laser requer um nível mínimo de transmissão para certa profundidade de tecido.

Através desse estudo, baseado em diferentes autores, pode-se chegar a uma conclusão para essa revisão bibliográfica. A tabela abaixo mostra os resultados obtidos no estudo do Laser na Acupuntura.

TABELA 1 - Estudos da literatura com o uso do Laser na Acupuntura

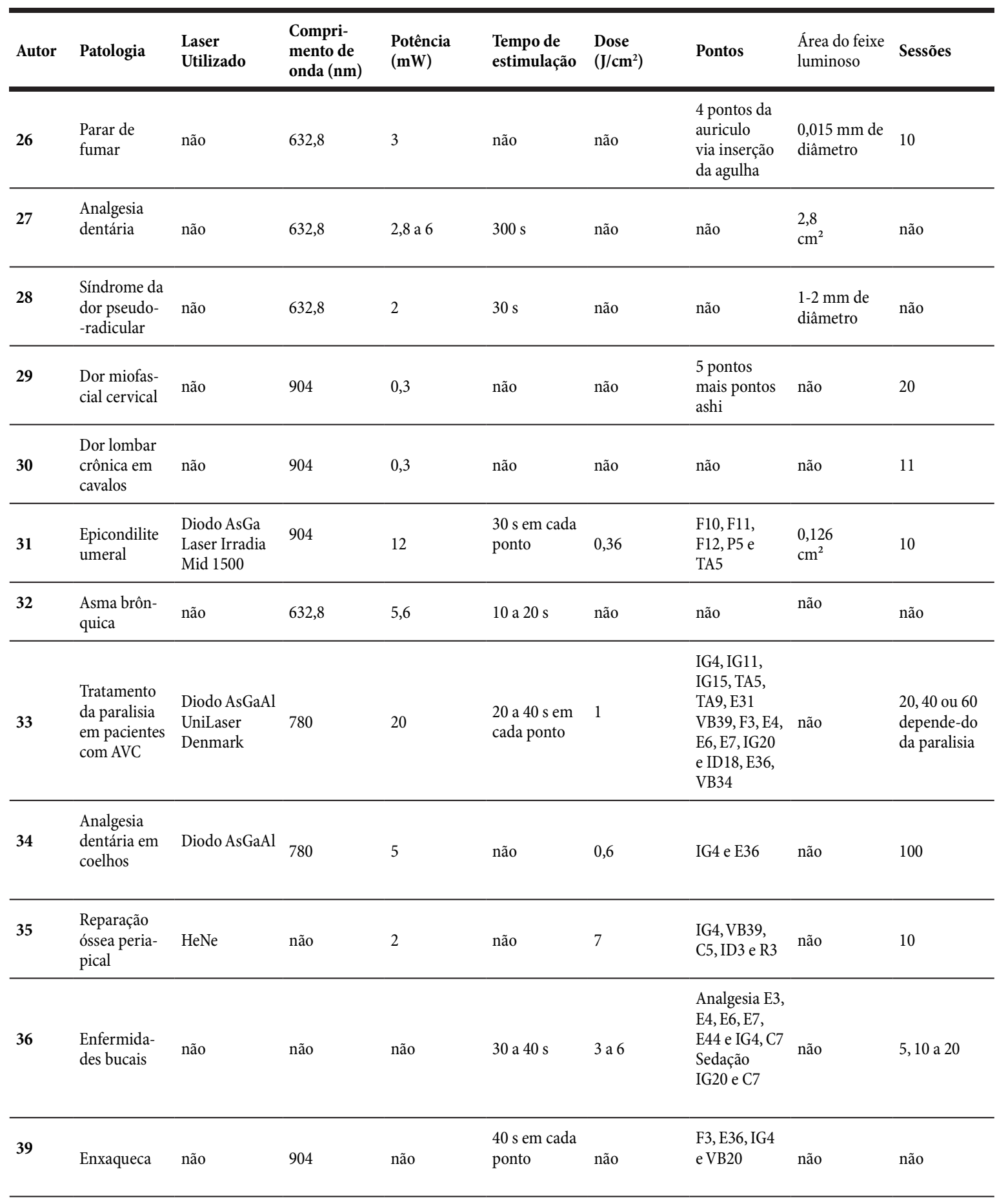




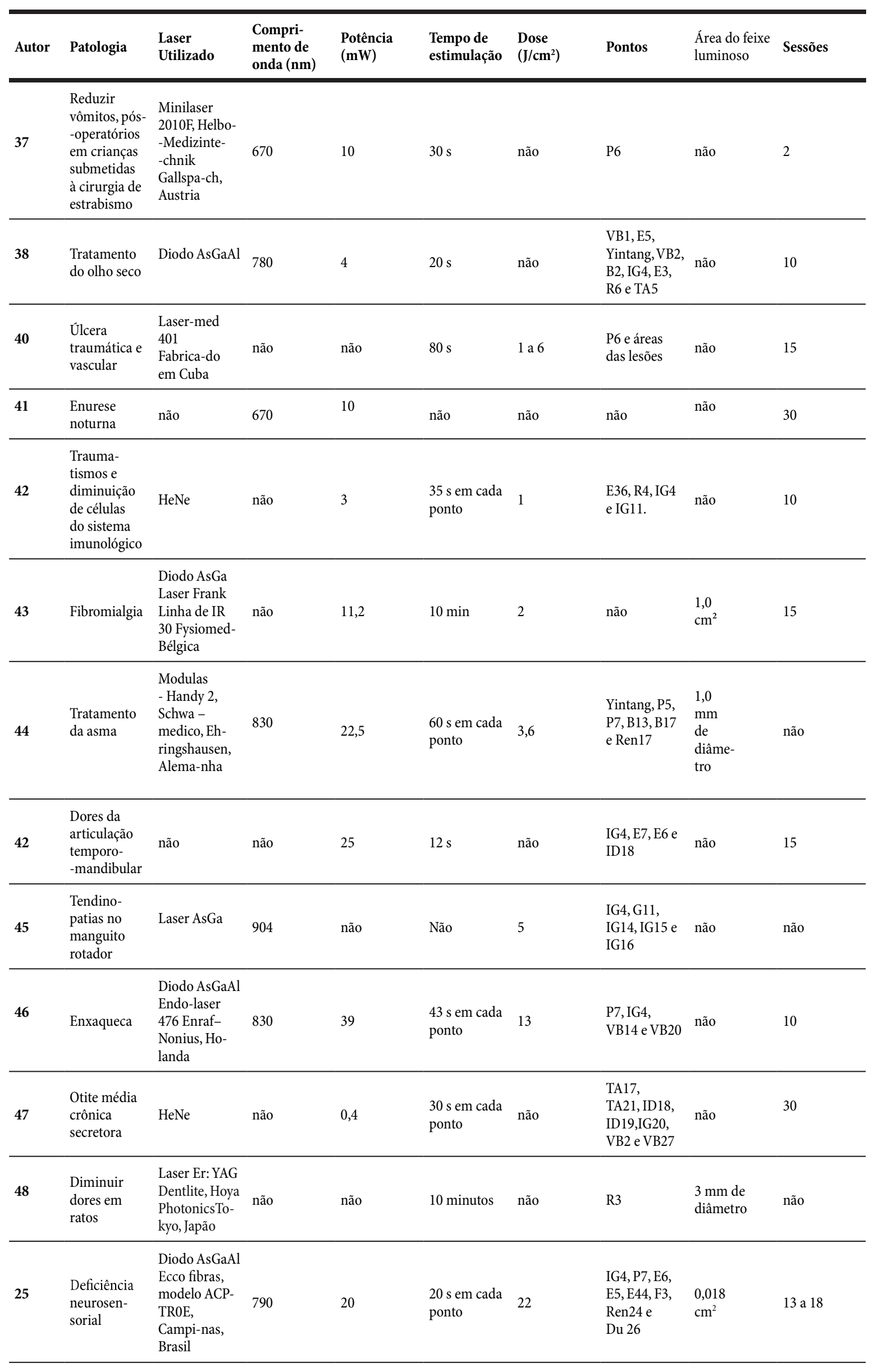




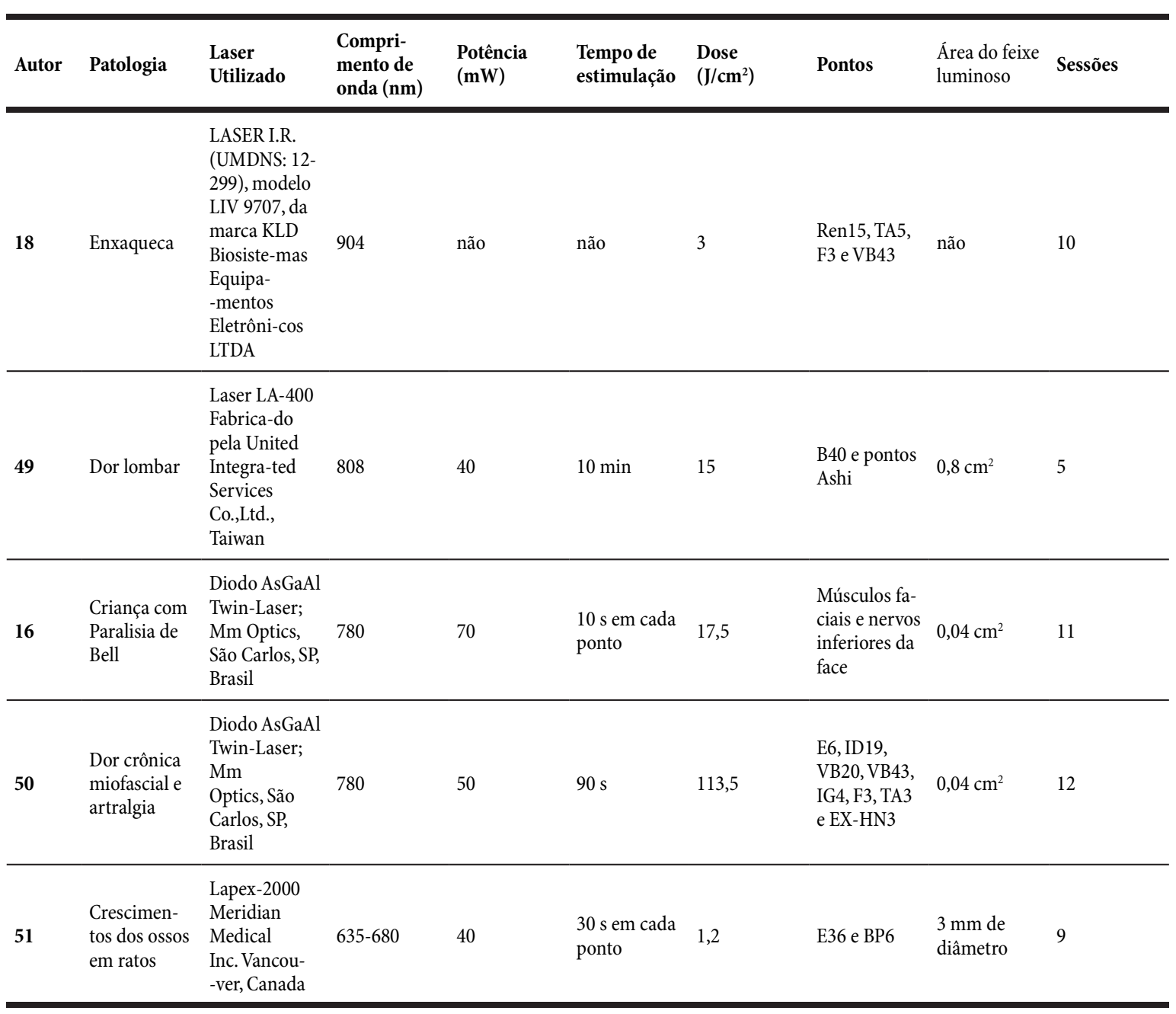

\section{CONSIDERAÇÕES FINAIS}

Durante essa revisão bibliográfica, verificou-se que a carência de material científico dificultou a elaboração, estruturação e enriquecimento da discussão e de tabelas sobre 0 assunto. Na literatura foi encontrado pouco material fundamentado sobre os estudos com Laseracupuntura, dificultando assim a padronização de protocolos com o uso de Laser na Acupuntura. Nesse contexto, evidencia-se a necessidade da continuidade de pesquisas envolvendo as variáveis em questão.

Por todas essas divergências apresentadas neste estudo ainda são necessárias muitas pesquisas e elaborações de protocolos para verificar qual o melhor tempo de estimulação em cada ponto, número de sessões, tempo de tratamento, comprimento de onda e dose a serem utilizadas para cada patologia.

Outro fator considerável e relevante para o emprego desta terapia é a qualificação do profissional e seu conhecimento sobre Acupuntura que envolvem o manuseio do equipamento a Laser e a escolha do melhor tratamento para atingir os resultados desejados, sem causar qualquer trauma ou riscos ao paciente.

\section{CONFLITOS DE INTERESSE}

declararam não haver

\section{FONTES DE FINANCIAMENTO}

nenhuma 


\section{REFERÊNCIAS}

1. Almeida-Lopes L. Laserpuntura: Bases Científicas e Aplicações[monografia de especialização].São Carlos: Escola Spaço Alternativo; 2010.

2. Brasil. Política Nacional de Práticas Integrativas e Complementares no SUS.Brasília: Ministério da Saúde, 2006.

3. Yamamura Y. Acupuntura tradicional: A arte de inserir. São Paulo: Roca, 1993.

4. Wang B. Princípios de medicina interna do Imperador Amarelo. São Paulo: Ícone, 2001

5. Maciocia G. Os fundamentos da medicina chinesa.2 ed. São Paulo: Roca, 2007.

6. Fröner TM. Laserpuntura. [trabalho de conclusão de curso].São Paulo: Universidade de São Paulo; 2007.

7. Goldman N,Chen M,Fujita T,Xu Q,Peng W,Liu W et al.Adenosine A1 receptors mediate local anti-nociceptive effects of acupuncture. NatureNeuroscience 2010; 3: 883-888.

8. Nogier PMP. Noções práticas de auriculoterapia.São Paulo: Andrei, 1998

9. Round R,Litscher G, Bahr F.Auricular Acupuncture with Laser.EvidenceBasedComplementaryandAlternative Medicine 2013; 2013: 1-22.

10. Draehmpaehl D,ZohmannA. Acupuntura no cão e no gato: princípios básicos e prática cientifica. São Paulo: Rocca, 1997.

11. Luna SPL. Emprego da acupuntura em anestesia. In: Fantoni, DT, Cortopassi SRG. Anestesia em cães e gatos. São Paulo: Roca; 2002. P 337-343.

12.Hayashi AM,Matera JM.Princípios gerais e aplicações da acupuntura em pequenos animais: revisão de literatura.Rev. Educ. Contin. CRMV.SP 2004; 8(2): 109-122.

13. Whittaker P. Laser acupuncture: past, present, and future. Lasers in Medical Science 2004; 19: 69-80.

14. Looney AL. Using acupuncture in veterinary practice. Veterinary Medicine 2000; 95: 615-619.

15. Chan WW, Chen KY, Liu H, Wu LS, Lin JH. Acupuncture for general veterinary practice. J. Vet. Med. Sci. 2001; 63: 1057-1062.

16. Fontana CR, Bagnato VS.Low-level laser therapy in pediatric Bell's palsy: case report in a three-year-old child. J. Altern. Complement. Med. 2013; 14(4); 376-382.

17. Scognamillo-Szabó MVR,Bechar, GH. Acupunture: Scientific basis and applications. Ciência Rural 2001; 31(6): 1091-1099.

18. Dias EC, Borges RF. O efeito da radiação Laser em $904 \mathrm{~nm}$ no tratamento de enxaquecas nos acupontos[trabalho de conclusão de curso]. Belém: Universidade da Amazônia; 2009.

19. Cruanes JC. La terapia laser, hoy. Barcelona: Centro de DocumentaciónLáserMeditec S.A.; 1984.

20. Machado MVM, Vulcano LC,Hussini CA, Alves ALG. Efeitos da Laser terapia em tendinite experimental no tendão flexor digital superficial em eqüinos: estudo histológico e ultra-sonográfico. ArchivesofVeterinary2000;5: 111-115

21. Cabrera EBA, Perón JMR, Alfonso LE. Laser Acupuntura con Hélio Neón en el tratamiento de pacientes traumatizados. Rev. Cubana Med. Milit.2002; 31(1): 5-12.

22. Anderson RR, Parrish JA. The optics of human skin. J. Invest Dermatol.1981; 77: 13-19.

23. VanGemert MJC, Jacques SL,Sterenborg HJCM, Star WM. Skin optics.IEEE Trans. Biomed.Eng. 1989; 36: 1146-1154.

24. Schieke SM, Schroeder P,Krutmann J. Cutaneous effects of infrared radiation: from clinical observations to molecular response mechanisms. PhotodermatolPhotoimmunolPhotomed 2003;19: 228-234.

25. Epelbaum E. Tratamento de Deficiência Neurosensorial por Laser em Baixa Intensidade e sua Associação a Acupuntura a Laser[dissertaçãode mestrado].São Paulo: Instituto de Pesquisas Energéticas e Nucleares; 2007.

26. Zalesskiy VN,Belousova IA, Frolov GV. Laser-acupuncture reduces cigarette smoking: a preliminary report. Acupunct.Electrother Res. 1983; 8: 297-302.

27. Zhou YC. An advanced clinical trial with laser acupuncture anesthesia for minor operations in the oromaxillofacial region. Lasers Surg. Med. 1984; 4: 297-303.
28. Kreczi T,KlinglerD. A comparison of laser acupuncture versus placebo in radicular and pseudoradicular pain syndromes as recorded by subjective responses of patients.Acupunct. Electrother Res. 1986; 11: 207-216.

29.Ceccherelli F,Altafini L, Lo Castro G,Avila A, Ambrosio F,Giron GP. Diode laser in cervical myofascial pain: a double-blindstudy versus placebo. Clin. J. Pain. 1989; 5: 301-304.

30. Kilde AM, Martin BB JR. Methods of stimulating acupuncture points for treatment of chronic back pain in horses. J. Am. Vet. Med. Assoc. 1989; 195: 1375-1379.

31. Haker E, Lundeberg T. Laser treatment applied to acupuncture points in lateral humeral epicondylalgia: a double-blind study. Pain 1990; 43: 243-247.

32. Tandon MK,Soh PFT, Wood AT. Acupuncture for bronchial asthma? A double-blind crossover study.Med. J. Aust. 1991; 154: 409-412.

33. Naeser MA, Alexander MP,Stiassney-Eder D,Galler V, Hobbs J, Bachman $D$ et al. Laser acupuncture in the treatment of paralysis in stroke patients: a CT scan lesion site study. Am. J.Acupunct. 1995; 23(1): 13-28.

34. Sing T, Yang MM. Electroacupuncture and laser stimulation treatment: evaluated by somatosensory evoked potential in conscious rabbits. Am. J. Chin. Med. 1997; 25: 263-271.

35. Andreu MIG, Zaldívar CJV. Empleo de la terapia láser en la reparación óssea periapical. Rev Cubana Estomatol 1997; 34(1).

36.Zaldívar CJV,Andreu MIG. Laser blando em puntos de acupuntura el tratamiento de enfermedades bucales. Rev. CubanaEstomatol 1997;34(1).

37. Schlager A, Offer T,Baldissera I. Laser stimulation of acupuncture point $\mathrm{P} 6$ reduces postoperative vomiting in children undergoing strabismus surgery. Br. J. Anaesth. 1998; 81: 529- 532.

38. Nepp J,Wedrich A,Akramian J,Derbolav A,Mudrich C,Ries E et al. Dry eye treatment with acupuncture: a prospective, randomized double-masked study. Adv. Exp. Biol. Med. 1998; 438: 1011-1016.

39. Lavies NG. Laser acupuncture for migraine and muscle tension headache: a double-blind controlled trial. Acupunct. Med. 1998; 16:73-76.

40. Morera EA, Álvarez EV, Ferrer MG,Méndez JH, Mora IT. Utilizacíon de laserpuntura em úlceras de miembros inferiores. RevistaCubana deCirurgia 2001; 40(2).

41. Radmayr C,Schlager A,Studen M,Bartsch G. Prospective randomized trial using laser acupuncture versus desmopressin in the treatment of nocturnal enuresis. Eur. Urol. 2001; 40: 201-205.

42. Cabrera KR et al. Radiación laser de baja potencia em el tratamiento del dolor disfunción de la A.T.M. Revista Avances 2003; 5(3).

43. Gür A,Karakoc M,Nas K,Cqevik R,Sarac J,Demir E. Efficacy of Low Power Laser Therapy in Fibromyalgia: A Single-blind, Placebocontrolled Trial. Lasers Med. Sci. 2002; 17: 57-61.

44. Gruber W,Eber E,Malle-Scheid D,Pfleger A, Weinhandl E,Dorfer L et al., Laser acupuncture in children and adolescents with exercise induced asthma. Thorax 2002; 57: 222-225.

45. Magalhães IH. O tratamento das tendinopatias do mangto rotador com laserterapia e laserpuntura[dissertação de mestrado]. Varginha: CentroUniversitário do Sul de Minas; 2005.

46. Ebneshahidi S,Heshmatipour M,Moghaddami A,Eghtesadiaraghi P. The effects of laser acupuncture in chronic tension headache: a randomized controlled trial. Acupuncture in Medicine 2005; 23(1): 13-18

47. Alvarez NP. Otitis Media Secretoria, tratamento com laser. Revista 16 de Abril Revista Estudiantil de las Ciencias Médicas 2006.

48. Zeredo JL, Sasaki KM, Toda K. High-intensity laser for acupuncture-like stimulation.Lasers Med. Sci. 2007; 22: 37-41.

49. Lin M, Wu H, Hsieh Y, Su C, Shih Y, Lin C et al. Evaluation of the Effect of Laser Acupuncture and Cupping with Ryodoraku and Visual Analog Scale on Low Back Pain. Evidence-Based Complementary and Alternative Medicine 2013;521617: 1-7.

50. Ferreira LA, Oliveira RG, Guimarães JP, Carvalho ACP, Paula MVQ Laser acupuncture in patients with temporomandibular dysfunction: a randomized controlled trial. Lasers Med. Sci. 2013; 28: 1549-1558.

51.Yeom M, Kim S, Lee B, Zhang X, Lee H,Hahm D et al.Effects of Laser Acupuncture on Longitudinal Bone Growth in Adolescent Rats. EvidenceBased Complementary and Alternative Medicine 2013; 424587: 1-7. 